\title{
Research Paper: Driver's Effective Factors in Traffic Accident: A Sequential Explanatory Mixed Methods crosshark Research Protocol
}

\author{
Azam Saei ${ }^{12^{*}}$, Ali Rahmani ${ }^{3}$,Abbas Ebadi ${ }^{4}$, Hamid Reza Khankeh $^{5}$ \\ 1. Behavioral Sciences Research Center, Faculty of Nursing, Baqiyatallah University of Medical Sciences, Tehran, Iran \\ 2. Department of Health, Rescue and Treatment of I.R. Iran Police Force, Applied Research Center, Tehran, Iran. \\ 3. Department of Military Nursing, Faculty of Nursing, Baqiyatallah University of Medical Sciences, Tehran, Iran. \\ 4. Department of Medical Surgery, Faculty of Nursing, Baqiyatallah University of Medical Sciences, Tehran, Iran. \\ 5. Department of Public Health Sciences, Karolinska Institutet, Stockholm, Sweden.
}

Article info:

Received: 30 Apr. 2017

Accepted: 03 Aug. 2017

\section{Keywords:}

Explanatory mixed methods, Human factors, Drivers, Traffic accidents
Citation: Saei A, Rahmani A, Ebadi A, Khankeh HR. Driver's Effective Factors in Traffic Accident: A Sequential Explanatory Mixed Methods Research Protocol. Health in Emergencies and Disasters Quarterly. 2017;3(1):31-38. https://doi.org/10.29252/ NRIP.HDQ.3.1.31

https://doi.org/10.29252/NRIP.HDQ.3.1.31

* Corresponding Author:

Azam Saei, PhD

Address: Behavioral Sciences Research Center, Faculty of Nursing, Baqiyatallah University of Medical Sciences, Tehran, Iran.

E-mail:saei.azam@yahoo.com 


\section{Introduction}

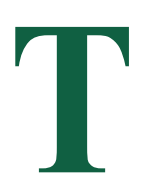

raffic accidents are listed in the top ten significant fatality causes in the world. It is a primary public health problem worldwide [1]. Annually, 1.2 million people die across the globe due to accident-related injuries and 20-50 million ones get injured [1, 2]. Studies showed that in 2000-2020, the number of accident victims decreases in Developed countries that is equivalent to $90 \%$ of the annual disability-Adjusted Life Years (DALYs) [3]. Consequently, it is estimated that traffic accidents which are at the eleventh position among the causes of death and injury will turn into the third health threat by 2020 [4].

In recent years, there has been a sudden increase in exposure to motor vehicles almost anywhere in the world, causing transport-related injuries (TRIs) the dominant mechanism of trauma in most parts of the world [5]. The global rank of our country, Iran, for accident-related mortality rate is eight while its position in the Eastern Mediterranean Region is two- the only second to Libya. The rate of traffic accidents in Iran is fifteen times more than in the developed countries [6], and hence, such accidents are a significant health problem in Iran [7]. The cost of road traffic injuries in Iran annually is about $2.19 \%$ of Gross Domestic Product [8], and hence, such accidents are a significant public health problem in Iran [7].

It has been estimated that human factors are the primary cause or a determining factor in $90 \%-95 \%$ of all traffic accidents. Part of the human factors includes physical, psychological, social, and cultural factors in drivers $[9,10]$. Physical factors significantly affect drivers' performance and the risk of accidents $[11,12]$. Some physical factors and illnesses which contribute to the incidence and the severity of traffic accidents include age [13-15], medications [14], fatigue and sleepiness [16], obesity [17], and chronic conditions like sleep apnea [18] and depression [19]. On the other hand, considerable stress and immobility also predispose drivers to diabetes mellitus $[20,21]$ and cardiovascular disease, accelerate their mortality rate, and affect the incidence of traffic accidents [22].

Besides, addictive substances, alcoholic drinks, and medications have been known to affect drivers' performance and the rate of traffic accidents [23]. Various studies have attempted to explain individual differences in risky driving behavior and traffic accident involvement through psychological issues [24]. Personality characteristics are associated with various risky driving behaviors, and crash risk [25]. Thus, it is crucial to identify and understand the factors that are involved in drivers' risky road behaviors. A Chinese report on driver safety defines that there are many factors which predict vehicle-driver collisions [26].

Since the variety of variables affect drivers' intention to use the road in risky situations, using a theoretical framework for the study of such variables would be crucial. The current study will use the Reason conceptual framework. Reason and his colleagues have developed a tool, i.e., Driver Behavior Questionnaire (DBQ), to measure and better understand these different behaviors among the drivers. According to them [27], unsafe behaviors could be categorized into two behavior divisions: violations and errors. They suggest that ill-suited driver behaviors ("violations," "errors" and "slips and lapses") will predict the intention to perform a given risky behavior [27].

The Swiss Cheese Model (SCM) is the most popular accident causation model and is used widely throughout various industries that proposed by Reason. Previous studies had shown that the SCM was a valid model in predicting multiple risky behaviors. All these latest research [28, 29] provide a deeper understanding of these actions and the factors explaining a different kind of drivers behaviors. The results showed that there was a significant correlation between the DBQ and drivers' risky behaviors. Although the most critical factor in predicting causes of drivers' behavioral patterns, research on accidents mainly focused on the road environment, driver and vehicles in the form of quantitative studies with little attention to qualitative studies.

Moreover, limited quantitative and even no qualitative studies have been conducted regarding this issue. Thus it is essential to conduct a study focusing on driver's behaviors, to provide a better understanding of drivers' risky behaviors. However, since the topic needs more in-depth information, we thought to conduct a study that provides both quantitative and qualitative data would be the best way to shade more light on this topic. Moreover, no studies have used the Reason's conceptual framework to explain drivers' intention in using the road in potentially risky situations in Iran where many drivers are causing death. These mixed methods study set out to determine traffic-risky behaviors in drivers as well as its determinants such as drivers' physical health, personality, driver behavior and socio-demographic characteristics and the Reason's conceptual framework's factors. Furthermore, we will explore drivers' perception of traffic-risky behaviors. Finally based on the findings, preventive and 
cultural based strategy for of accidents due to the driver will be proposed.

The aim of this mixed methods study is to determine driver's traffic-risky behaviors, physical health, and personality. Furthermore, driver's experience of behaviors will be explored. On the basis of the findings, comprehensive and culturally sensitive strategies for promoting the safety of Iranian driver will be developed.

The specific objectives of this study are: 1 . To determine the drivers' traffic-risky behaviors in using the roads; 2 . To determine the drivers' physical health in using the roads; 3 . To determine the drivers' personality in using the roads; 4 . To determine the association between drivers' physical health and drivers' traffic-risky behaviors; 5. To determine the association between drivers' personality and drivers' traffic-risky behaviors; 6. To explore the perception drivers of all ages and level of experiences about the traffic-risky behaviors in using the roads; and 7. To offer strategies for modifying traffic-risky behaviors of Iranian drivers of all ages and level of experiences.

\section{Materials and Methods}

The present study is designed as sequential explanatory mixed methods approach valuing both objective and subjective information. The 'follow-up explanation model' adopted to explain the quantitative results by collecting qualitative data [30]. The explanatory sequential design has two phases. During the first phase, the researcher will identify specific quantitative results that need additional explanation in quantitative data. The second, qualitative phase will be designed based on the first phase to explain the quantitative unexpected findings that need additional explanations. Then, the researcher gathers qualitative data from those participants who can explain these unexpected findings adequately.

The merging of results occurs in the interpretation and explanation of the quantitative and qualitative results [31]. The quantitative data would provide a general understanding of factors of traffic-risky behaviors in drivers of all ages and level of experiences, and the qualitative data would explore participants' views about the problem perfectly therefore, it could be a rational design (Figure 1).

\section{Phase one: Quantitative study}

This strand is designed as a population-based crosssectional study to assess offending risky drivers in using the road in a representative sample of Iranian population of all ages and level of experiences (18-60) living in Tehran, the capital of Iran.

\section{Sample size and sampling method}

The target population is drivers of all ages and level of experiences, male and female living in Tehran. The required sample size was calculated based on the Cochrane formula of the driver's risky behaviors in previous studies $(\mathrm{d}=0.05, \mathrm{z}=1.96, \mathrm{P}=0.64)$. As per the calculation, approximately 360 drivers will be required for this study. To select a typical sample, a simple random sampling was carried out. Every household within 22 different districts in Tehran would have the same probability of being sampled. A convenient sample of 360 drivers was recruited from among traffic offenders who had referred to the Center for Seized Cars, driver clinics, insurance, and traffic police Tehran. After obtaining the informed consent, the participants answered the questionnaire individually.

\section{Eligibility criteria}

The inclusion criteria were drivers residing in Tehran, aged 18-60, Iranian nationality, could speak Persian, did not suffer from cognitive disorders, agreed to participate in the study.

\section{Scales and data collection}

The questionnaire comprising four sections was completed through a face-to-face interview. It consists of questions on sociodemographic characteristics, the Driver Medical Questionnaire (DMQ), the NEO-Five Factor Inventory Questionnaire and the Driver Behavior Questionnaire (DBQ).

\section{Questions on sociodemographic characteristics}

The first section consists of 14 questions about socio demographic characteristics including age, gender, occupation, marital status, driving experience, traveled distance in an annual, road accident record (considering the time), etc.

\section{Driver Medical Questionnaire (DMQ)}

Section 2 consists of 17 questions. One of the screening tools for identifying drivers and license applicants' health problems is the DMQ. The DMQ is a simple, short, and easily-applicable instrument which assesses the most significant physical and mental health problems affecting traffic accidents in all ages [32]. DMQ was translated into Persian, and after cultural accommodation, it was 
re-evaluated by two other professional translators; then its validity and reliability were approved. We performed pre-testing to confirm item clarity. The questionnaire was delivered to 45 drivers, and they were asked to answer the questions and identify the questions which are not clear. Afterwards, some minor changes were made to some questions. The questionnaire was delivered to 5 police-doctors to adapt it with our country checkups and to confirm. To identify internal reliability, internal consistency analysis (Kuder-Richardson 21 formula) and testretest for the data from the pilot study were measured.

Accordingly, 45 traffic offenders were invited to complete the Persian DMQ twice with a fourteen-day interval. Then, the Intraclass Correlation Coefficient (ICC) was calculated between the scores obtained from two measurement time points. The Kr21 value for the DMQ was 0.68 . Kr21 values of greater than 0.7 are considered satisfactory. Besides, the ICC between the scores of the two measurement time points was 0.948 . ICC values are interpreted as poor $(0-0.20)$, fair $(0.21-0.40)$, moderate (0.41-0.60), good (0.61-0.80), and very good (0.81-1).

\section{NEO-Five Factor Inventory Questionnaire}

Section 3 consists of 60 questions. A measure of personality survey (NEO-Five Factor Inventory) was administered.

\section{Driver Behavior Questionnaire (DBQ)}

Section 4 comprises 50 questions. Subjects are asked to answer the questions by a simple analysis and identify how often they commit abnormal behaviors during driving. It is widely used today to gather ill-suited driver behaviors ("violations," "errors" and "slips and lapses") declared by individuals.

\section{Data analyses}

Descriptive statistics was used to explore the quantitative data. Data were analyzed by SPSS (V. 23) using chi-square and Pearson correlation coefficient. Multivariable linear regression analysis will be applied to predict the impact of each independent variables (sociodemographic variables and Driver Behavior) on the dependent variable (NEO-Five Factor Inventory and health problems) and determine the variance.

Phase two: Qualitative study

Sampling method

A purposive sampling strategy will be conducted to explain quantitative unexpected findings.

\section{Data collection}

Data was collected via individual in-depth, semistructured, face-to-face interviews. The research team reviews the questions, and the ways to gather valid data and focus on research questions was dome before conducting the interviews. Interviews were started with one open question and continued by in-depth questions using a pre-established interview guide held.

In case of requirement, focus group discussions were conducted. Before any interviews, we supplied recording equipment. The data was recorded through tape recording and note-taking. Interviews were taped and written on a piece of the paper word by word after the interview. Interviews were continued until data saturation, and no new themes related to the phenomenon under study emerge.

\section{Data analyses}

Data were analyzed using the content analysis approach. It is a professional technique to process scientific data in a structured fashion. The taped interviews were written down on a piece of the paper word by word and read for several times to acquire a general sense of data. The document was condensed into units. The units were coded and divided into themes and sub-themes regarding similarities and differences to reveal drivers (of all ages and level of experiences) perceptions of traffic-risky behaviors in using the road. During the interviews, the data will be recorded through note taking and tape recording.

Data collection was continued until saturation is reached and no new themes emerge in the interviews. In this study, peer and member reviewing was used to achieve reliability and was performed to assess the trust worthiness of the data. Qualitative data was managed by using the MAXQDA10 software.

\section{Integration of quantitative and qualitative data}

Due to the sequential and explanatory design of the study, we analyzed the quantitative and qualitative components separately because the qualitative phase depends on the quantitative phase [33]. Then, qualitative and quantitative phases were integrated to display how they are related to each other.

\section{Ethics approval}

The study design and objectives were thoroughly explained to subjects. Also, subjects will be free to quit at any time. Written informed consent was taken from 


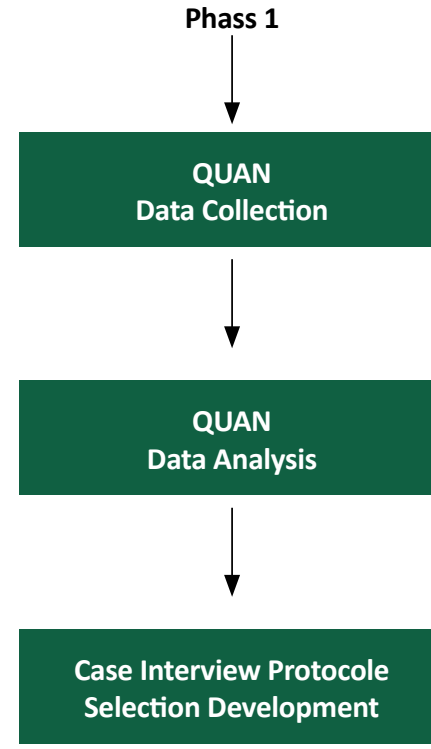

Phass 2
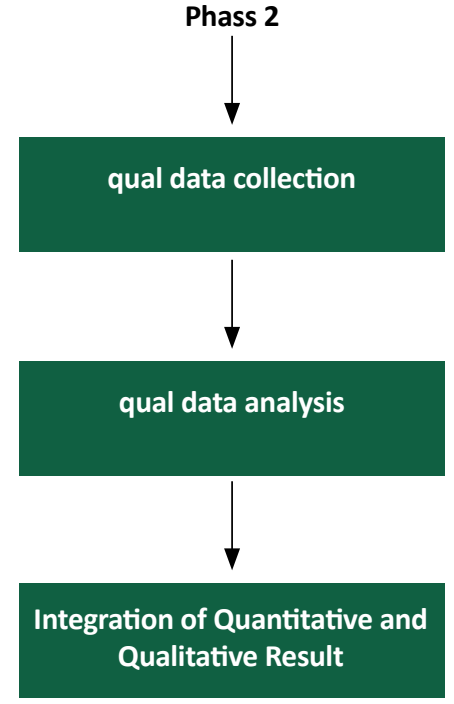

Procedure

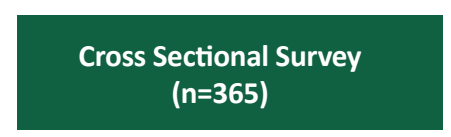

SPSS Software

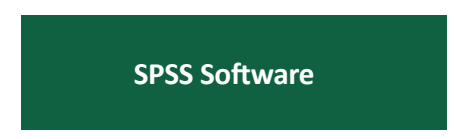

Purposefully Selecting Participants Based on Maximal Variation
Product

Numeric Data
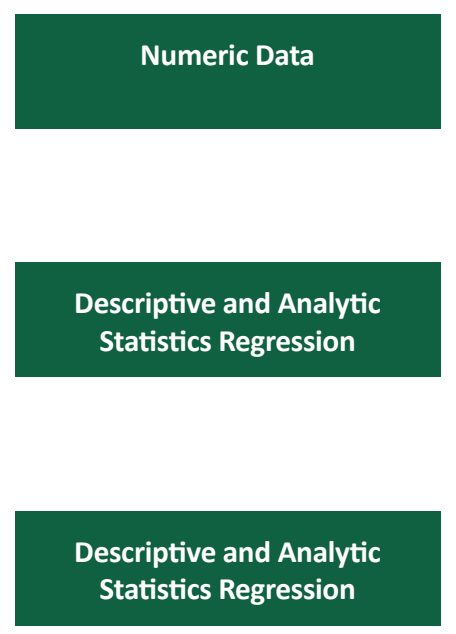

Interview Topic Guide

Developing Interview Questions
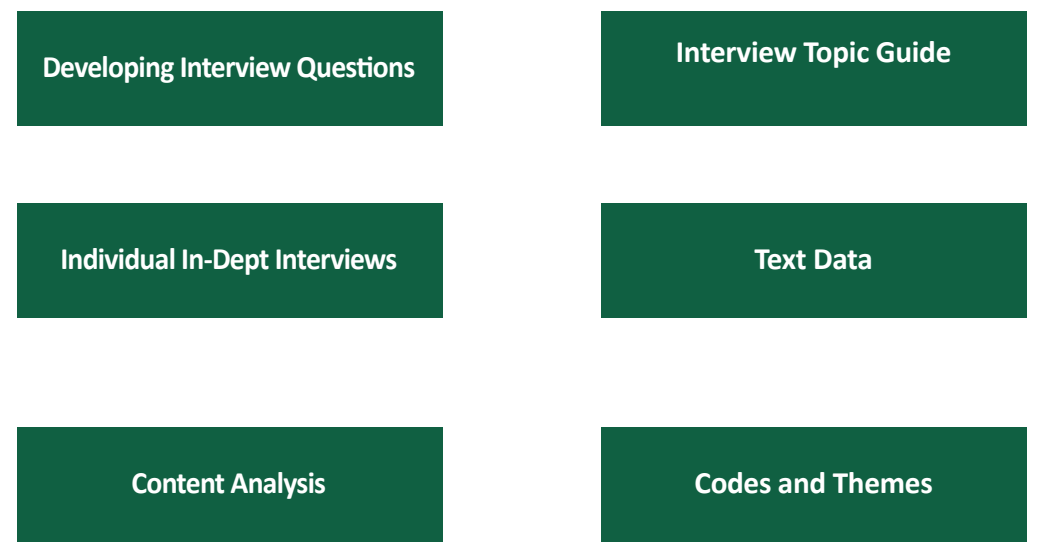

Interpretation and Explantation Quantitative

Codes and Themes
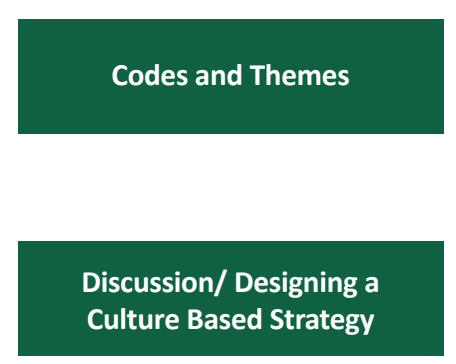

Hlealth in

Emergencies and D]isasters [Oluarterly

Figure 1. The study framework derived from Creswell et al. [31]

all participant before the study commenced. The ethics approval of this study has been obtained by the Ethics Committee of Baqiyatallah University of Medical Sciences, Tehran, Iran (code number: 93-02-28-10902).

\section{Discussion}

Traffic accidents and injury prevention is a primary global concern [3]. Drivers need to have an acceptable level of physical and mental health, and psychological well-being to reduce risky road behaviors [34]. This study will benefit from some characteristics that make it possible. First, the location of the study is in a very active center (the Center for Seized Cars) with a tremendous potential for recruitment in both strands of the study. Second, the main strength of this study will provide a better understanding of the topic, information and robust data on driver's factors for risky behaviors through a culturally sensitive approach. The collection of both quantitative and qualitative Strand, allow us to understand the reasons, perceptions of drivers about their risky behaviors in potentially dangerous situations. Third, a review of the related literature on risky behaviors and the focus group discussion among experts will be applied, so it would be possible to develop strategies for promoting health behaviors, the qualitative and quantitative results. 
It is assumed that results of this study will be revealed comprehensive necessary information about the drivers' traffic-risky behaviors, which improving the prevention insight, education of drivers, and seeking the support of police officer.

\section{Conclusion}

The findings of this explanatory mixed methods study will provide information on traffic-risky behaviors by all drivers. The results will be implemented to design a cultural based strategy, and intervention programs are to be designed to promote driver's health in the community. This study also will provide some insights into the health behavior factors that need to be considered if effective strategies and intervention programs are to be designed to promote driver's health in Iran .The results of this paper will be disseminated as peer-reviewed publications at conferences and as part of a doctoral thesis.

\section{Acknowledgements}

This research was financially supported by Traffic Police Research Center. This article was part of a study conducted in Baqiyatallah University of Medical Sciences, Iran. This is a study protocol of the first Author at Faculty of Nursing, Baqiyatallah University of Medical Sciences, Tehran, Iran. Ali Rahmani and Abbas Ebadi are joint supervisors for the study. Hamid Reza Khankeh was the study advisor. All authors are contributed to all aspect of the investigation. All of the authors critically evaluated the paper and provided the final draft.

\section{Conflict of Interest}

The authors declared no potential conflicts of interest concerning the research, authorship, or publication of this article.

\section{References}

[1] World Health Organization. Global status report on road safety 2013: Supporting a decade of action. Geneva: World Health Organization; 2013.

[2] World Health Organization. Global status report on road safety: Time for action. Geneva: World Health Organization; 2009.

[3] Leandro M. Young drivers and speed selection: A model guided by the Theory of Planned Behavior. Transportation Research Part F: Traffic Psychology and Behaviour. 2012; 15(3):219-32. doi: 10.1016/j.trf.2011.12.011
[4] World Health Organization. World report on road traffic injury prevention. Geneva: World Health Organization; 2004.

[5] Leproust S, Lagarde E, Salmi LR. Systematic screening for unsafe driving due to medical conditions: Still debatable. BMC Public Health. 2008; 8(1). doi: 10.1186/1471-2458-8-27

[6] Moafian G, Aghabeigi MR, Hoseinzadeh A, Lankarani KB, Sarikhani Y, Heydari ST. An epidemiologic survey of road traffic accidents in Iran: Analysis of driver related factors. Chinese Journal of Traumatology. 2013; 16(3):140-4

[7] Ardalan A, Masoomi GR, Goya MM, Sarvar MR, Haddadi M, Miadfar J, et al. Road traffic injuries: A challenge for Iran's health system. Iranian Journal of Public Health. 2009; 38(1):98101.

[8] Rezaei S, Arab M, Karami Matin B, Akbari Sari A. Extent, consequences and economic burden of road traffic crashes in Iran. Journal of Injury and Violence Research. 2014; 6(2). doi: 10.5249/jivr.v6i2.191

[9] Karacasu M, Er A. An analysis on distribution of traffic faults in accidents, based on driver's age and gender: Eskisehir case. Procedia-Social and Behavioral Sciences. 2011; 20:776-85. doi: 10.1016/j.sbspro.2011.08.086

[10] Shappell SA, Wiegmann DA. Human factors investigation and analysis of accidents and incidents. Encyclopedia of Forensic Sciences. 2013; 440-9. doi: 10.1016/b978-0-12-3821652.00146-x

[11] Sagberg F. Driver health and crash involvement: A case-control study. Accident Analysis \& Prevention. 2006; 38(1):28-34. doi: 10.1016/j.aap.2005.06.018

[12] Classen S, Crizzle AM, Winter SM, Silver W, Eisenschenk S Evidence-based review on epilepsy and driving. Epilepsy \& Behavior. 2012; 23(2):103-12. doi: 10.1016/j.yebeh.2011.11.015

[13] Borowsky A, Shinar D, Oron Gilad T. Age, skill, and hazard perception in driving. Accident Analysis \& Prevention. 2010; 42(4):1240-9. doi: 10.1016/j.aap.2010.02.001

[14] Clarke DD, Ward P, Bartle C, Truman W. Older drivers' road traffic crashes in the UK. Accident Analysis \& Prevention. 2010; 42(4):1018-24. doi: 10.1016/j.aap.2009.12.005

[15] Duke J, Guest M, Boggess M. Age related safety in professional heavy vehicle drivers: A literature review. Accident Analysis \& Prevention. 2010; 42(2):364-71. doi: 10.1016/j. aap.2009.09.026

[16] Jayatilleke AU, Nakahara S, Dharmaratne SD, Jayatilleke AC, Poudel KC, Jimba M. Working conditions of bus drivers in the private sector and bus crashes in Kandy district, Sri Lanka: A case control study. Injury Prevention. 2009; 15(2):80-6. doi: 10.1136/ip.2008.018937

[17] Anderson JE, Govada M, Steffen TK, Thorne CP, Varvarigou $\mathrm{V}$, Kales SN, et al. Obesity is associated with the future risk of heavy truck crashes among newly recruited commercial drivers. Accident Analysis \& Prevention. 2012; 49:378-84. doi: 10.1016/j.aap.2012.02.018

[18] Rodenstein D. Sleep apnea: Traffic and occupational accidents \& ndash; Individual risks, socioeconomic and legal implications. Respiration. 2009; 78(3):241-8. doi: $10.1159 / 000222811$ 
[19] Hilton MF, Staddon Z, Sheridan J, Whiteford HA. The impact of mental health symptoms on heavy goods vehicle drivers' performance. Accident Analysis \& Prevention. 2009; 41(3):453-61. doi: 10.1016/j.aap.2009.01.012

[20] Izadi N, Malek M, Aminian O, Saraei M. Medical risk factors of diabetes mellitus among professional drivers. Journal of Diabetes \& Metabolic Disorders. 2013; 12(1):23. doi: $10.1186 / 2251-6581-12-23$

[21] Graveling AJ, Frier BM. Driving and diabetes: Problems, licensing restrictions and recommendations for safe driving. Clinical Diabetes and Endocrinology. 2015; 1(1). doi: 10.1186/ s40842-015-0007-3

[22] Roth GA, Forouzanfar MH, Moran AE, Barber R, Nguyen $\mathrm{G}$, Feigin VL, et al. Demographic and epidemiologic drivers of global cardiovascular mortality. New England Journal of Medicine. 2015; 372(14):1333-41. doi: 10.1056/nejmoa1406656

[23] Li G, Brady JE, Chen Q. Drug use and fatal motor vehicle crashes: A case control study. Accident Analysis \& Prevention. 2013; 60:205-10. doi: 10.1016/j.aap.2013.09.001

[24] Ulleberg P, Rundmo T. Personality, attitudes and risk perception as predictors of risky driving behaviour among young drivers. Safety Science. 2003; 41(5):427-43. doi: 10.1016/s0925-7535(01)00077-7

[25] Ehsani JP, Li K, Simons Morton BG, Tree McGrath CF, Perlus JG, O'Brien F, et al. Conscientious personality and young drivers' crash risk. Journal of Safety Research. 2015; 54:83e2987. doi: $10.1016 /$ j.jsr.2015.06.015

[26] Yang J, Du F, Qu W, Gong Z, Sun X. Effects of personality on risky driving behavior and accident involvement for chinese drivers. Traffic Injury Prevention. 2013; 14(6):565-71. doi: 10.1080/15389588.2012.748903

[27] Reason J, Manstead A, Stradling S, Baxter J, Campbell K. Errors and violations on the roads: A real distinction. Ergonomics. 1990; 33(10-11):1315-32. doi: 10.1080/00140139008925335

[28] Özkan T, Lajunen T, Summala H. Driver behaviour questionnaire: A follow-up study. Accident Analysis \& Prevention. 2006; 38(2):386-95. doi: 10.1016/j.aap.2005.10.012

[29] Aberg L, Rimmo PA. Dimensions of aberrant driver behaviour. Ergonomics. 1998; 41(1):39-56. doi: 10.1080/001401398187314

[30] Baheiraei A, Mirghafourvand M, Mohammadi E, Nedjat S, Charandabi SMA, Rajabi F, et al. Health promoting behaviors and social support of women of reproductive age, and strategies for advancing their health: Protocol for a mixed methods study. BMC Public Health. 2011; 11(1). doi: 10.1186/14712458-11-191

[31] Creswell JW, Klassen AC, Plano Clark VL, Smith KC. Best practices for mixed methods research in the health sciences. Bethesda (Maryland): National Institutes of Health; 2011.

[32] Drivers Medical Group. At a Glance: Guide to the current medical standards of fitness to drive. Swansea: Drivers Medical Group; 2010.

[33] Teddlie C, Tashakkori A. Foundations of mixed methods research: Integrating quantitative and qualitative approaches in the social and behavioral sciences. California: Sage Publication; 2009.
[34] Meuser TM, Berg Weger M, Niewoehner PM, Harmon AC, Kuenzie JC, Carr DB, et al. Physician input and licensing of at-risk drivers: A review of all inclusive medical evaluation forms in the US and Canada. Accident Analysis \& Prevention. 2012; 46:8-17. doi: 10.1016/j.aap.2011.12.009 
